\title{
Neural Net-Based Anomaly Detection System in Substation Networks
}

\author{
Philipp Kreimel \\ Limes Security \\ Hagenberg, Austria \\ www.limessecurity.com \\ pkr@limessecurity.com
}

\author{
Paul Tavolato \\ University of Applied Sciences \\ St. Pölten \\ Institute of IT Security Research \\ St. Pölten, Austria \\ www.fhstp.ac.at \\ paul.tavolato@fhstp.ac.at
}

\begin{abstract}
Important components of the electric energy distribution systems are primary and secondary substations. Due to the incorporation of legacy communication infrastructure in these systems, they often have inherent cyber-security vulnerabilities. Further, traditional intrusion defence strategies for IT systems are often not applicable. In order to improve cyber-security in substation networks, this paper presents a neural net-based monitoring system. Further, to evaluate the applicability of the system, all experiments were conducted on a real test bed, which represents the substation domain as close as possible to reality. The proposed monitoring system covers several tasks. First, relevant network packets are acquired from network traffic and analysed. Based on these packets statistical features are extracted. Then, classes are defined, and a normal behaviour model of the network is trained by the neural net. New network traffic is compared to the model, in order to determine the nature of the traffic and identify potential anomalies. Finally, the monitoring system is evaluated by conducting several supervised and unsupervised network attacks against the test bed.
\end{abstract}

Substation, Electric Power Systems, Anomaly Detection, IEC 60870-5-104, IEC 61850, Neural Network

\section{INTRODUCTION}

Implementing smart grid concepts necessarily requires the integration of information and communication technologies into the systems of production, distribution, and usage of electric energy. This makes cyber security a crucial new topic in this area as the number of potential attack points of the distribution network for electrical energy rises considerably. Local attacks are no more restricted to the actual attack point but can spread over the whole net. In the past, strongly networked systems in critical infrastructures, especially in connection with the electrical power system, have been subject to cyber-attacks (see for example the reports of the US ICS-CERT - Industrial Control Systems Cyber Emergency Response Teams (US-CERT 2019)). Such infrastructures are rewarding targets for groups with criminal or terroristic intentions.

Important components of the electric energy distribution systems are primary and secondary substations. Within these substations implementing the smart grid leads to a high degree of automation which results in increased use of data processing technologies and hence, opens up a huge number of hitherto unknown security problems. The main reason for this is the integration of modern communication technologies such as microprocessor-based intelligent electronic devices (IEDs), the use of standard protocols like TCP/IP, Ethernet, and connections to WANs by Internet technologies. Remote access to IEDs or to the internal user interface of the communication infrastructure, for purposes of (remote) maintenance, are quite common nowadays. Using insecure standard protocols opens up a dangerous potential for attacks having serious consequences for the whole infrastructure.

Common security solutions are usually limited to regulating the access to the components as a whole (firewalls, encryption and other ways of perimeter security); they are used in substations, too. But their efficiency is limited in this application area because they were originally designed for conventional IT systems and are not suited for the special requirements of the hardware and software architectures of substations. Hence, they are not sufficient: we urgently need a second line of defence when an attacker conquers this first line of perimeter security. We propose to use a system that continuously monitors the behaviour of the network. A model of the normal 
behaviour of the network traffic in a substation is constructed by machine-learning methods and later on used during operation to detect anomalous behaviour. Such security solutions for substations are still in a preliminary state as argued in (Hong et al. 2012).

The rest of the paper is organised as follows. Section 2 gives an overview of related work. In Section 3, we describe the characteristics of substations. In Section 4, the methodology is defined. Section 5 presents the neural net-based monitoring system. Section 6 describes experimental classification results. Section 7 concludes the paper.

\section{RELATED WORK}

Security topics of SCADA-based automation networks in substations is discussed in several papers on a general level, e.g. in (Zhang et al. 2019), (Kwon et al. 2019), (Premaratne et al. 2010), (Rashid et al. 2014), (Oman et al. 2000), (Dzung et al. 2005). So far, mainly security measures known from conventional information and communications systems have been transferred to SCADA environments, such as Intrusion Detection Systems. There are some works for the special area of substations.

\subsection{Model-Based Approaches}

In (Cheung et al. 2007) the authors implement a model-based IDS in a process control system. They use the Modbus protocol with TCP/IP, but the authors claim that the same method can be used with other process protocols, too. They investigate three different methods for model-based detection of security breaches, starting from the characterization of Modbus TCP and the design of Snort rules. At the next level, the communication patterns among the network components are described. At the third level, a learning-based model can compute probabilities of abnormal behaviour by using a Bayes-network.

Hahn et al. (Hahn and Govindarasu 2013) use a model-based approach, too, to construct MINDS (Model-based Intrusion Detection for the Smart grid), an IDS with three components. The first component contains a model of the substation together with all devices and communication described by Petri-Nets. Detection is based on comparisons of the monitored network traffic with the Petri-net model. The results of this detection procedure are then used as input for further analysis on system level, to identify coordinated complex attacks as well.

Barbosa and Pras (Barbosa and Pras 2010) describe an anomaly detection method based on the analysis of network data streams. Data is collected in periodic intervals in order generate models in an ongoing way and to use these models for anomaly detection.

In (Yang et al. 2014) the authors present a stateful IDS for IEC 60870-5-104 SCADA networks. The IEC/104 protocol is modelled as a deterministic finite state automaton (for those parts for which this is possible). The advantages of this whitelisting method is its capability to detect hitherto unknown attacks, too.

\subsection{Statistical Approaches}

A paper by Düssel et al. (Düssel et al. 2009) proposes an anomaly detection method based on the payload and operating in real time. The system defines a property space by looking at the distribution of each n-th byte of the TCP payload data and detects abnormal packets by comparing them to normal byte sequences. This technique may be used for upper level protocols by looking at n-grams of the TCP payload.

In (Yoo and Shon 2015) the authors developed a method of anomaly detection for MMS (Manufacturing Message Specification) and GOOSE (Generic Object Oriented Substation Events), the two main components of the IEC 61850 protocol. The first step, preprocessing, consists of three phases. Packets are filtered, relevant information is extracted and combined with information from related packets to build information streams. Additionally, traffic-based data sets are generated, which shall detect Denial-of-Service attacks by observing transfer rates. All the collected data is used as training data in Expectation-Maximization Clustering (EM) and Local Outlier Factor (LOF) algorithms to eliminate outliers. To train normal behaviour Yoo and Shon use Support Vector Machine (SVM) algorithms. During monitoring MMS- and GOOSE-packets from the log files are compared with the learned SVM model and added to the training data set to improve the model.

In (Yang et al. 2006) information from network traffic and statistical data from the hardware is used to identify deviations from normal behaviour by methods of pattern matching. Their experiments show, that auto associative kernel regression together with SPRT (Statistical Probability Ratio Test) can detect deviating behaviour in SCADA environments.

In (Premaratne et al. 2008) the authors use network data to distinguish between normal user behaviour and downloading of malicious data by Support Vector Machine and Nearest Neighbors Classifiers. Sosha et al. (Shosha et al. 2011) propose a distributed intrusion detection system based on sensors. The system consists of several local detection agents and global detection agents, which work together to detect anomalies by correlating 
data from the components.

\subsection{Signature-Based Approaches}

Yang et al. (Yang et al. 2013) describe a rule-based IDS for SCADA networks using the IEC 60870-5104 protocol. It contains signature-based methods for misuse detection and model-based methods to describe expected behaviour.

Ten et al. (Ten et al. 2011) describe anomaly detection by looking at four parameters: 1) attack attempts; 2) changes in the file system; 3) changes in the configuration of the target system; 4) changes of the system state in the target system. Using these four attributes, they claim, malicious behaviour could be detected on all levels of a substation and a footprint of this behaviour could be generated.

The authors in (Yang et al. 2013) suggest signaturebased methods as well as model-based methods. By applying signature-based rules, in some cases even the origin of a threat or attack could be located. The model-based part uses a whitelisting method to guard against unknown attacks.

\section{CHARACTERISTICS OF SUBSTATIONS}

The architecture of a substation shows a local network connected to the - potentially insecure - outside WAN by a router; this router is usually secured by conventional means of perimeter security. The local network is subdivided in two parts: the guard net containing the IEDs to safeguard the energy distribution net against defective electrical issues like short-circuit faults or ground faults; and the automation network connecting the IEDs and the RTUs (remote terminal units) and providing services for the automated operation of the substation. These two parts are mainly working independently from each other but are connected physically within the communication network.

\subsection{Transmission Protocols}

Currently, several open international standards exist in SCADA systems of the electrical power, such as Distributed Network Protocol Version 3 (DNP3) (IEEE 2010), IEC 60870-5 series (IEC 2006), and IEC 61850 (IEC 2013). In this paper we focus on the latter two, as they are mainly used in Europe.

All protocols are TCP/IP- or Ethernet-based. The use of standard protocols and the connection of these local networks to potentially insecure networks outside results in various security problems. Further, the protocols transmit messages in clear text without any form of authentication. Therefore, systems are susceptible for network-based attacks such as Manin-the-Middle and protocol-specific attacks.

\section{METHODOLOGY}

As mentioned above there is an urgent need for innovative approaches to design a second line of defence that is not restricted to measures of perimeter security. From a security point of view, the automation network is a crucial point in the architecture as it is reachable from outside (for remote maintenance or similar services) and on the other hand it is connected to the guard net. We propose a monitoring solution for primary and secondary substations. Devising this solution as an anomaly detection system will enable it to cope with hitherto unknown, not yet observed attacks as well. As network traffic in mentioned components of the electricity distribution system shows rather regular behaviour, anomaly-centred procedures are viable. The monitoring system uses machine-learning techniques to construct a model of the normal behaviour of the automation network in a substation and in a second stage uses this model to monitor network behaviour permanently. In case of a detected anomaly, a first classification of the anomaly is undertaken, and an alarm is raised.

\subsection{Test System}

Our goal was to implement and test the monitoring system on a test system that could represent a real substation facility. We used a test system provided by our project partner Siemens, which incorporates multiple RTUs, a running logic and data transmission using the IEC 104 and the IEC 61850 protocols. Through this system a realistic implementation as well as a real network environment was available as a test bed.

\section{NEURAL NET-BASED MONITORING SYSTEM}

In order to detect anomalies in substation networks, we present a neural net-based monitoring system. First, the system acquires training data from a presumably attacker-free substation network and trains a model of valid behaviour. Then, new network traffic is compared with the model to detect possible anomalies.

To test the applicability of our system, we developed a prototype implementation on a Raspberry $\mathrm{Pi} 3$ and integrated it into the previously mentioned test system. The setup and the workflow of the monitoring system is shown in Figure 1. The components and the operation of the system is described in detail in the following Sections.

\subsection{Data Acquisition}

The first procedure of the monitoring system is the acquisition of data, in this case network packets of the substation devices. All relevant data from the 


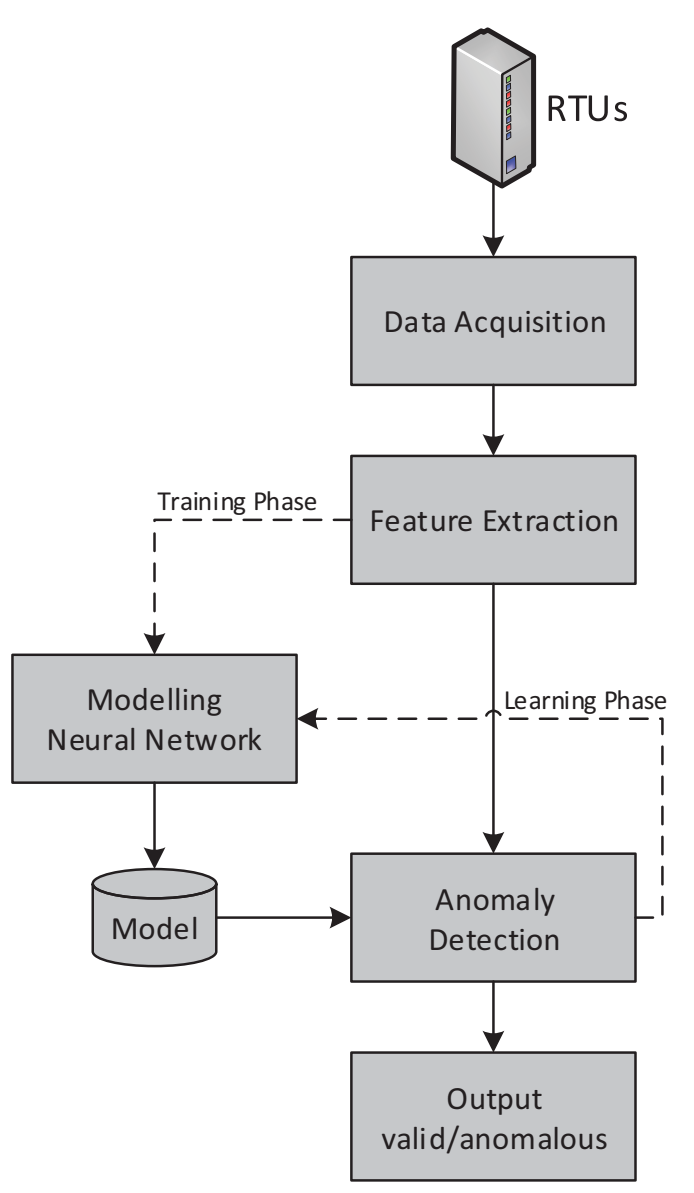

Figure 1: Concept of the neural net-based monitoring system

devices should be acquired in real-time, to allow on the fly monitoring of the network traffic.

This was achieved by configuring a mirror port on the test system's firewall, which in this case also acts as a local switch for all connected devices in the test network. The mirror port forwards a copy of all network packets from a designated port, the gateway through which all traffic runs, to our monitoring system. This provides real-time capturing of the entire network traffic, both incoming and outgoing. Further, we developed a custom-built packet capture filter for substation protocols, which is based on the Pcap4J library (Yamada 2019). The captured network traffic, and the resulting data set, contains protocol-specific data of the respective configured protocol (IEC 60870-5-104 or IEC 61850) as well as relevant network packet information.

\subsection{Feature Extraction and Selection}

The recorded network packets from the data acquisition process are presented in raw format. This does not allow for easy processing by a machine learning algorithm. Therefore, we use feature extraction to extract informative and comparable features from the network packets. In addition, feature extraction allows to reduce the dimensionality of data, while still maintaining all relevant information.

Feature extraction is often a difficult task, requiring detailed understanding of the protocols and other domain-specific knowledge, as only a specific set of features with significant information should be extracted. The following features were extracted from the network packets.

- Round-trip-time (RTT);

- Packet length;

- Packets per second;

- TCP window size;

- Measurement values sent by the protocols;

- Communication paths $(\operatorname{SrcIP}->\operatorname{DstIP})$.

Selecting features is one of the most important as well as the most difficult tasks in anomaly detection. With too few features information can be lost, with too many there is a risk of overfitting. In the course of the project, we tested various features for their information gain. We discovered that extracting features from single network packets does not produce suitable information for anomaly detection. Further analyses of the network traffic showed that a cycle-based approach, i.e. calculation of statistical features on a certain number of packets, yields more promising results. This will be discussed in the following Section.

\subsection{Valid Behaviour Model}

The first step in creating a model of valid behaviour of the test system is the training of valid data to describe the normal state of the devices to be trained. In this case, two RTUs. We tested various approaches for training a valid behaviour model based on the network traffic. However, the asynchronous structure and nature of the two transmission protocols in use (IEC 60870-5-104 and IEC 61850) was a challenge:

(i) Measured values are only sent if values change.

(ii) Network packets cannot be classified individually due to the asynchronous transmission.

During the analysis of network traffic, it has been recognized that over a certain period of time, specific transmissions are performed periodically and thus classes (e.g. RTUs), can be uniquely identified. From this, a possible solution of the aforementioned problems was derived: Based on a defined number of packets features are calculated and trained for the 
respective class. For our test system this range was defined with $k=50$, which extracts features from the last 50 packets acquired for this specific class. For this specific set we calculate statistical features standard deviation, see Equation 1, and mean, see Equation 2, - for each of the extracted features. This identifies periodic patterns in the data set, which allows the data to be trained by a classifier.

$$
\begin{gathered}
\sigma=\sqrt{\frac{1}{N-1} \sum_{i=1}^{N}\left(x_{i}-\bar{x}\right)^{2}} \\
\bar{x}=\frac{1}{n}\left(\sum_{i=1}^{n} x_{i}\right)=\frac{x_{1}+x_{2}+\cdots+x_{n}}{n}
\end{gathered}
$$

From our test system we collected 400 instances from each of the RTUs and built a data set of valid behaviour. Based on this data set, a model was designed by experimenting with different classifiers. In order to check the accuracy of the classifier a 10-fold cross-validation was used. In addition, the performance (calculation effort and duration) of the classifiers was considered. We experimented with several classifiers, such as $k$-nearest neighbors algorithm, SVM, naive Bayes classifier and neural networks. The highest accuracy, as shown in Table 1, was achieved with a neural network. We used a feed-forward neural network trained by a backpropagation algorithm (multi-layer perceptron). The model generated by this classifier provides the basis for the classification of unknown data.

Table 1: Confusion matrix of the valid behaviour model

\begin{tabular}{ccl}
\hline $\mathrm{a}$ & $\mathrm{b}$ & $<-$ classified as \\
\hline 389 & 11 & $\mathrm{a}=$ rtu20_valid \\
6 & 394 & $\mathrm{~b}=$ rtu30_valid \\
\hline
\end{tabular}

\subsection{Anomaly Detection}

To test the model in a supervised environment, various network attacks were executed under supervision, allowing for various evaluations. It should be noted, that the focus of most attacks was to be as covert as possibly, i.e. modifying protocol variables only slightly, in order to cause unknown system states and stay undetected for a longer period. The attacks also show the inherent security issues of substation protocols, as discussed in Section 3.1. The following substationspecific network attacks against the IEC 60870-5104 protocol were executed:

(i) Man-in-the-Middle filter attack, which constantly overwrites transmitted ASDU values sent from a specific IOA number.

(ii) Man-in-the-Middle increment attack, which slightly modifies transmitted ASDU values (value $+0.1-1.0)$. Through this attack, unknown system states could be caused.

(iii) Man-in-the-Middle drop attack, which discards packets that send a specific signal.

(iv) Denial-of-Service attack using hping 3 against the web server of the RTU. However, this attack was not trained in the model, as the RTUs contain security measures which causes it to stop sending any communication packets. Thus, no packets during this attack could be acquired.

From the resulting data set, the neural network was re-trained to classify the supervised attacks as well. The overall performance of the classifier was again evaluated with a cross-validation. The classification results of the supervised learning is shown in Table 2. An overall classification accuracy of $91.5 \%$ could be achieved, with high class precision and class recall across most classes. Both the filter and drop attack could be classified with high accuracy. Only the increment attack was partly misclassified, which is shown by the low class recall and precision, approx. $36 \%$ and $76 \%$, respectively. This can be attributed to the fact that the packet modifications during this attack were minimal and thus difficult to identify.

\section{DETECTING ANOMALIES IN UNSUPERVISED DATA}

In order to evaluate the statistical performance of the monitoring system, we tested the system against unsupervised network traffic. As described in Section 5, the system acquires live network traffic, extracts features out of the packets and applies this data against the trained model. The classifier will calculate a class prediction based on confidences towards a certain class.

The tested instances contain several network attacks, both trained and untrained, as well as valid instances. The result of the classification process is shown in Table 3. The Table lists the class prediction and the respective confidences, as well as the true class label - which is unknown to the classifier to identify the attacks. The results look promising, as most instances were classified correctly and with satisfactory accuracy. For certain classes, the calculated confidences are well over $98 \%$.

However, certain instances, such as the MitM increment attacks (rows 9 to 11), were not classified distinctively. This results from the hidden nature of this attack, as protocol values are modified only marginally. Thus, this network traffic is similar to valid traffic and the confidences for valid classes are higher.

The results achieved by our monitoring system 
Table 2: Confusion matrix of the supervised attacks

\begin{tabular}{lcccccl}
\hline classified as $->$ & $\mathrm{a}$ & $\mathrm{b}$ & $\mathrm{c}$ & $\mathrm{d}$ & $\mathrm{e}$ & class precision \\
\hline $\mathrm{a}=$ rtu20 valid & 380 & 12 & 0 & 2 & 0 & $96.45 \%$ \\
$\mathrm{~b}=$ rtu30 valid & 17 & 380 & 0 & 49 & 1 & $85.01 \%$ \\
$\mathrm{c}=$ rtu30 mitm-filter & 0 & 0 & 136 & 0 & 0 & $100.00 \%$ \\
$\mathrm{~d}=$ rtu30 mitm-incr & 2 & 7 & 0 & 29 & 0 & $76.32 \%$ \\
$\mathrm{e}=$ rtu30 mitm-drop & 1 & 1 & 0 & 0 & 67 & $97.10 \%$ \\
\hline class recall & $95.00 \%$ & $95.00 \%$ & $100.00 \%$ & $36.25 \%$ & $98.53 \%$ & \\
\hline
\end{tabular}

are promising. Various unknown substation-specific attacks could be detected with high accuracy.

\section{DISCUSSION}

In dealing with security challenges in substations, our approach was that a monitoring system could be an applicable second line of defence. In order to show the viability of anomaly detection in substation networks, all experiments were conducted on a test bed with substation components, which represents a realistic implementation as well as a real network environment.

The proposed monitoring system covers several tasks. First, relevant network packets are acquired from network traffic. Based on these packets statistical features are calculated. Then, classes are defined, and a normal behaviour model of the network is trained by the neural net. New network traffic is compared to the model, in order to determine the nature of the traffic.

To test the detection rate of our system, several substation-specific network attacks were conducted against the test bed under supervision. An overall classification accuracy of $91.5 \%$ could be achieved for these attacks. Additionally, the detection rate of unsupervised attacks also yielded promising results. The results show that network-based attacks in substation networks can be detected with high confidence using the monitoring system. The experiments were conducted against the test bed, which demonstrates the applicability in realistically simulated substation networks and had the advantage that attacks could be realized, too, in order to generate anomalous data showing effects of cyber-attacks. The system consists of a machinelearning component and a monitoring component. It is implemented on a Raspberry $\mathrm{Pi}$ and can easily be integrated into a substation needing only some preliminary configuration work to adapt it to the specifics of the substation under consideration. First tests in a real operational substation were conducted and showed satisfactory performance, too. Though it was not possible to conduct proper cyber-attacks in this real-life setting, at least some extraordinary switching and similar activities were performed to generate anomalous situations. The system detected all these anomalies correctly. Further work will comprise tests in substations of different sizes and different configurations.

\section{ACKNOWLEDGEMENTS}

This research was funded by the Austrian Climate and Energy Fund and supported by our project partners Siemens AG Austria and Wels Strom $\mathrm{GmbH}$.

\section{REFERENCES}

Barbosa, R. R. R. and A. Pras (2010). Intrusion detection in scada networks. In IFIP International Conference on Autonomous Infrastructure, Management and Security, pp. 163-166. Springer.

Cheung, S., B. Dutertre, M. Fong, U. Lindqvist, K. Skinner, and A. Valdes (2007). Using modelbased intrusion detection for scada networks. In Proceedings of the SCADA security scientific symposium, Volume 46, pp. 1-12. Citeseer.

Düssel, P., C. Gehl, P. Laskov, J.-U. Bußer, C. Störmann, and J. Kästner (2009). Cybercritical infrastructure protection using real-time payload-based anomaly detection. In International Workshop on Critical Information Infrastructures Security, pp. 85-97. Springer.

Dzung, D., M. Naedele, T. P. Von Hoff, and M. Crevatin (2005). Security for industrial communication systems. Proceedings of the IEEE 93(6), 1152-1177.

Hahn, A. and M. Govindarasu (2013). Model-based intrustion detection for the smart grid (minds). In Proceedings of the Eighth Annual Cyber Security and Information Intelligence Research Workshop, pp. 27. ACM.

Hong, J., A. Stefanov, C.-C. Liu, and M. Govindarasu (2012). Cyber-physical security in a substation. In 2012 IEEE Power and Energy Society General Meeting, pp. 1-1. IEEE.

IEC (2006). Telecontrol equipment and systems-part 5-104: Transmission protocols-network access for 
Neural Net-Based Anomaly Detection System in Substation Networks Kreimel • Tavolato

Table 3: Classification results on unknown data

\begin{tabular}{lllllll}
\hline true label & $\begin{array}{l}\text { conf } \\
\text { rtu20_valid }\end{array}$ & $\begin{array}{l}\text { conf } \\
\text { rtu30_valid }\end{array}$ & $\begin{array}{l}\text { conf } \\
\text { mitm-filter }\end{array}$ & $\begin{array}{l}\text { conf } \\
\text { mitm-incr }\end{array}$ & $\begin{array}{l}\text { conf } \\
\text { mitm-drop }\end{array}$ & label prediction \\
\hline rtu-172-16-1-129-valid & 0,981 & 0 & 0 & 0,018 & 0 & rtu20_valid \\
rtu-172-16-1-129-valid & 0,989 & 0 & 0 & 0,011 & 0 & rtu20_valid \\
rtu-172-16-1-129-valid & 0,998 & 0 & 0 & 0,002 & 0 & rtu20_valid \\
rtu-172-16-2-1-mitm-drop & 0 & 0,02 & 0 & 0 & 0,979 & rtu30_mitm-drop \\
rtu-172-16-2-1-mitm-drop & 0 & 0,013 & 0,005 & 0 & 0,981 & rtu30_mitm-drop \\
rtu-172-16-2-1-mitm-drop & 0,001 & 0,009 & 0 & 0 & 0,987 & rtu30_mitm-drop \\
rtu-172-16-2-1-mitm-incr & 0 & 0,001 & 0,001 & 0,997 & 0 & rtu30_mitm-incr \\
rtu-172-16-2-1-mitm-incr & 0 & 0,001 & 0,007 & 0,992 & 0 & rtu30_mitm-incr \\
rtu-172-16-2-1-mitm-incr & 0 & 0,383 & 0 & 0,617 & 0 & rtu30_mitm-incr \\
rtu-172-16-2-1-mitm-incr & 0 & 0,306 & 0 & 0,693 & 0 & rtu30_mitm-incr \\
rtu-172-16-2-1-mitm-incr & 0,549 & 0,269 & 0 & 0,181 & 0 & rtu20_valid \\
\hline
\end{tabular}

iec 60870-5-101 using standard transport profiles. vol. IEC, 60870-5.

IEC (2013). Communication networks and systems for power utility automation. vol. IEC, 61850-1.

IEEE (2010, July). leee standard for electric power systems communications - distributed network protocol (dnp3). IEEE Std 1815-2010, 1-775.

Kwon, Y., S. Lee, R. King, J. I. Lim, and H. K. Kim (2019). Behavior analysis and anomaly detection for a digital substation on cyber-physical system. Electronics 8(3), 326.

Oman, P., E. Schweitzer, and D. Frincke (2000). Concerns about intrusions into remotely accessible substation controllers and scada systems. In Proceedings of the Twenty-Seventh Annual Western Protective Relay Conference, Volume 160. Citeseer.

Premaratne, U., J. Samarabandu, T. Sidhu, B. Beresh, and J.-C. Tan (2008). Evidence theory based decision fusion for masquerade detection in iec61850 automated substations. In 2008 4th International Conference on Information and Automation for Sustainability, pp. 194-199. IEEE.

Premaratne, U., J. Samarabandu, T. Sidhu, R. Beresh, and J.-C. Tan (2010). Security analysis and auditing of iec61850-based automated substations. IEEE Transactions on Power Delivery 25(4), 2346-2355.

Rashid, M. T. A., S. Yussof, Y. Yusoff, and R. Ismail (2014). A review of security attacks on iec61850 substation automation system network. In Proceedings of the 6th International Conference on Information Technology and Multimedia, pp. 510. IEEE.

Shosha, A. F., P. Gladyshev, S.-S. Wu, and C.-C. Liu (2011). Detecting cyber intrusions in scada networks using multi-agent collaboration. In 2011 16th International conference on intelligent system applications to power systems, pp. 1-7. IEEE.

Ten, C.-W., J. Hong, and C.-C. Liu (2011). Anomaly detection for cybersecurity of the substations. IEEE Transactions on Smart Grid 2(4), 865-873.

US-CERT (2019, May). ICS-CERT Control Systems Advisories and Reports. https://ics-cert.uscert.gov.

Yamada, K. (2019, May). Pcap4J - A Java library for capturing, crafting, and sending packets. https://www.pcap4j.org/.

Yang, D., A. Usynin, and J. W. Hines (2006). Anomaly-based intrusion detection for scada systems. In 5th intl. topical meeting on nuclear plant instrumentation, control and human machine interface technologies (npic\&hmit 05), pp. 12-16.

Yang, Y., K. McLaughlin, T. Littler, S. Sezer, B. Pranggono, and H. Wang (2013). Intrusion detection system for iec 60870-5-104 based scada networks. In 2013 IEEE power \& energy society general meeting, pp. 1-5. IEEE.

Yang, Y., K. McLaughlin, T. Littler, S. Sezer, and H. Wang (2013). Rule-based intrusion detection system for scada networks. Renewable Power Generation Conference (RPG 2013).

Yang, Y., K. McLaughlin, S. Sezer, Y. Yuan, and W. Huang (2014). Stateful intrusion detection for iec 60870-5-104 scada security. In 2014 IEEE PES General Meeting - Conference \& Exposition, pp. 1-5. IEEE.

Yoo, H. and T. Shon (2015, Jan). Novel approach for detecting network anomalies for substation automation based on iec 61850. Multimedia Tools and Applications 74(1), 303-318. 
Zhang, J., L. Jun'e, C. Xiong, N. Ming, W. Ting, and L. Jianbo (2019). A security scheme for intelligent substation communications considering real-time performance. Journal of Modern Power Systems and Clean Energy, 1-14. 